\title{
Eye contact, pupil dilation, and personal preference
}

JOHN W. STASS AND FRANK N. WILLIS, JR.

UNIVERSITY OF MISSOURI AT KANSAS CITY

Subjects were asked to choose partners for experiments. The available partners differed in eye-contact or in pupil dilation. Subjects of both sexes were more likely to choose partners with eye-contact during an introduction. Males were more likely to choose female available partners with dilated pupils. Similarly females chose male partners with dilated pupils.

The importance of visual contact in the interaction between animals has been described for many species in a variety of settings. The present study deals with the importance of visual contact for humans in their choice of partners for a future interaction.

Visual contact is not only important to human interaction but is also capable of being detected with extreme accuracy. Argyle \& Dean (1965) investigated eye-contact and found that without it Ss did not feel that they were fully in communication. Gibson \& Pick (1963) found that considerable accuracy marks the reception of a glance from another at an angle of 70 degrees.

Exline $(1960,1963,1965)$ has shown that eye contact in interaction is related to affiliation, sex, topic of conversation, and role as speaker or listener.

A second area of visual communication that has received some attention is the effect of pupillary size. Hess (1965) reported that an increase in pupil size was related to interest in a viewed object. He found further that males were more attracted to a picture of a female with dilated pupils that they were to an identical picture with undilated pupils.

There were two purposes for the present study. The first was to determine the effect of eye-contact on the choice of partners for a future interaction. The second was to examine the effect of pupil size on personal preference in an actual interaction as opposed to the response to photos as investigated by Hess.

\section{Subjects}

The study was conducted in three phases. The first dealt with eye-contact and employed 37 male and 35 female Ss. The second dealt with pupil dilation and employed 54 male Ss. The third dealt with pupil size and employed 19 female Ss. All Ss were undergraduate students in three colleges in the greater Kansas City area.

\section{Apparafus}

Pupillary dilation was achieved with a drug containing neo-synephrine $10 \%$ hydrochloride in a buffered low surface tension vehicle with sodium phosphate, sodium biphosphate and an antiseptic preservative, Zephiran chloride 1:10,000. This drug was selected because of its rapid effect, prolonged action, and lack of undesirable systemic side effects.

\section{Procedure}

The Ss were told that they were to take part in an experiment that required an experimental partner. The Ss in phase I were told that the experiment involved the handling of money and that they should choose a partner they could trust. Ss in phases II and III were told that the experimental task required very intimate communication and that they should choose someone who was, in their opinion, pleasant and easy to talk to. Each $S$ was taken to a room in which two available experimental partners were waiting. Ss were told that they would be introduced to two possible partners and that they were to inform $\mathrm{E}$ privately following the introduction as to their choice of partner. The available partners varied as follows: In phase I, one individual maintained eyecontact throughout the introduction while the other glanced away from the $S$ during the introduction. In this phase both partners were male. They were similarly dressed. Their position in relation to the Ss was rotated (left-right) for each $\mathrm{S}$. The partner giving eye-contact was rotated every fourth $\mathrm{S}$. All partners in all phases were rehearsed by $\mathrm{E}$ so that their smile, casual posture, etc. were similar during the introduction. In phase II it was considered important that the partners be approximately equal in attractiveness, so two women were chosen from a group of 12 because they were rated as being most equal in attractiveness by a class of over 200 introductory psychology students. On the first day on which phase II was run, one of the two women had dilated pupils, and on the second day the other had dilated pupils. The dilation was obvious enough to permit identification of the dilated partner at a distance of four feet. An optometrist administered the drug and checked the size of pupils at $10-\mathrm{min}$. intervals throughout the experimental period. No changes in pupil size were noted after the original dilation. In phase III, procedures were identical to phase II except that male identical twins were employed as partners. The men and women serving as available experimental partners were not informed of the nature of the study until all Ss were run. All Ss were asked to give reasons for their choice of partners following the session. Results

Phase I. 58 Ss chose the eye-contact partner and 14 chose the non-eye-contact partner. A chi-square test of goodness of fit yielded a $\mathrm{x}^{2}=26.8$, with $\mathrm{df}=1$ and $\mathrm{p}$ $<.01$. The proportion of male and female Ss choosing the eye-contact partner were approximately equal. 
The men serving under the two conditions were chosen about equally often.

Phase II. 36 Ss chose the dilated partner and 18 chose the non-dilated partner. A chi-square test of goodness of fit yielded a $x^{2}=6$, with $d f=1$, and $p<$ .02. The two women chosen as available partners did not appear to be equally attractive to the Ss as planned since one was chosen twice as often as the other, but each girl was chosen approximately twice as often in the dilated condition as in the non-dilated condition.

Phase III. 21 Ss chose the dilated partner and five chose the non-dilated partner. This resulted in a $X^{2}=9.8$, with $d f=1$, and $p<.01$. The men serving as available partners were chosen about equally often.

Reasons given for choice of partners. Only eight of the $72 \mathrm{Ss}$ in phase I mentioned eye-contact as being important in their choice. Other Ss stated that the partner appeared to be friendly, pleasant, nice, etc. In phases II and III, no S reported a choice based upon pupil size. Ss either stated that they were unable to explain their choice or simply stated that their choice was good looking, appeared friendly, pleasant, etc.

\section{Discussion}

All of the data from the present study support the generalization that visual contact may be quite important in forming initial impressions. This seems to be reflected in every day language in such expressions as "an honest face" and "shifty eyes." In view of Hess' finding that dilated pupils are an expression of interest, this study is significant in showing that women, like men, are attracted by others who appear to be interested in them.
A lack of eye contact may, in our culture, be interpreted as an indication of dishonesty or may, in the words of Argyle \& Dean (1965) indicate that channels for communication are closed.

It is interesting that although Ss may have a preference for others depending upon the appearance or behavior of the other's eyes, the Ss are not necessarily able to report the use of these cues.

In spite of the training and rehearsal of the partners aimed at controlling all behavior and appearance other than those being varied, it is possible that other differences existed at the time of the choice and influenced the choice. It should be noted, however, that the partners were not informed of the relationship being investigated, nor did they express an awareness of these relationships after the experiment was concluded. One might reason that an eyecontact partner could guess that he was to appear more attractive, but it does not follow that the partner in the dilated condition would make a similar guess.

\section{References}

Argyle, M., \& Dean, J. Eye-contact, distance, and affiliation. Sociometry, 1965, 28, 289-304.

Exline, R. v. Effects of sex, norms, and affiliative motivation upon accuracy of interpersonal preferences. J. Pers., 1960, 28, 397412.

Exline, R. V. Explorations in the process of persons perception: Visual interaction in relation to competition, sex, and need for affiliation. J. Pers., 1963, 31, 1-20.

Exline, R. V., et al. Visual behavior in a dyad as affected by interview content and sex of respondent. J. Pers. soc. Psychol., $1965,1,201-209$.

Gibson, J. J., \& Pick, A. D. Perception of another's looking behavior. Amer. J. Psychol., 1963, 76, 386-394.

Hess, E. H. Attitude and pupil size. Scient. American, 1965, 212, 46-54. 